\title{
On Extending Coverage of UMTS Networks Using an Ad-Hoc Network with Weighted Fair Queueing*
}

\author{
R. El-Azouzi ${ }^{1}$, R. El-Khoury ${ }^{1}$, A. Kobbane ${ }^{1,2}$, and E. Sabir ${ }^{1,2}$ \\ ${ }^{1}$ LIA/CERI, Université d'Avignon, Agroparc BP 1228, Avignon, France \\ ${ }^{2}$ LIMIARF, Université Mohammed V Rabat-Agdal, \\ 4 Av. Ibn Battouta B.P. 1014, Maroc \\ \{rachid.elazouzi, ralph.elkhoury, abdellatif .kobbane, \\ essaid.sabir\}@univ-avignon.fr
}

\begin{abstract}
In this paper, we are interested in the interconnection between an ad-hoc network and UMTS system. In presence of two access technologies, new challenges arise and mobile host will have face multiple base stations or gateways with different utilizations. As a result, a user who needs to establish a voice call, prefers to be connected through UMTS and a user who needs to transmit a Best Effort traffic with high rate, prefers to be connected through ad-hoc network. The aim of that combination is to improve the coverage of cellular networks and increases the services which can be made available for mobiles. This combination also reduces transmission power for mobile hosts. Our main result is characterization of stability condition and the end-to-end throughput using the rate balance. Numerical results are given and support the results of the analysis. Finally, we perform extensive simulation and verify that the analytical results closely match the results obtained from simulations.
\end{abstract}

Keywords: Interconnection, cross-layer, routing, ad-hoc, UMTS, stability.

\section{Introduction}

In order to meet the increasing in data and user mobility, a considered amount of effort is tunneled towards the convergence of multi-service networks of diverse technologies. Next generation wireless networks (NGWN) will be heterogeneous radio access technologies such UMTS, WiMAx, WLAN, etc, coexist. However, the NGWN will be a joint radio resource management among multiple operators of different radio resource technologies [3. In this paper, we are interested in the planning of ad-hoc networks in providing an improved cellular coverage of UMTS cellular networks and different services can be made available for nodes. Indeed, there exist several types of traffic that require different QoS. In presence of several access technologies, new challenge arises and mobile host will

\footnotetext{
* This work was partially supported by ANR WINEM No. 06TCOM05 and Maroc

Telecom R\&D No. 10510005458.06 PI.
} 
face multiple base stations or gateway with different utilization. This opens the possibility of using the most appropriate multiple access types for each type of call. For example, for a network with a low bandwidth and high reliability access as UMTS, it is preferable to be used by a voice call. As a result, a user prefers to be connected through UMTS network which can offer higher reliability for particular services, even at a high cost.

A multi-hop wireless ad hoc network is a collection of nodes that communicate with each other without any established infrastructure or centralized control. Many factors interact with each other to make the communication possible like routing protocol and channel access. Recently, wireless ad-hoc networks have been envisioned of commercial applications such as providing Internet connectivity for nodes that are not in transmission range of a wireless access point. Cellular network and ad-hoc networks should be considered as complementary systems. Hence, it is possible to use ad-hoc networks as an extension of the cellular networks like UMTS. Thus it is possible for a multihomed node to use different access technologies at different time to assure a permanent connectivity. As a result, potential benefits can be envisaged for UMTS cellular as : extend the cellular coverage, reduce the power transmission which implies the reduction of intra-cell and extra-cell interference. In the ad-hoc networks, new services can be made available as VoIP and streaming video.

In this work we are interested in the interconnection between a MANET and a $3 \mathrm{G}$ system. In order to be able to connect to more than one base station (or network), a terminal should have at least two network cards. In the ad-hoc network, we consider the random access mechanism for the wireless channel where the nodes having packets to transmit in their transmit buffers attempt transmissions by delaying the transmission by a random amount of time. This mechanism acts as a way to avoid collisions of transmissions of nearby nodes in the case where nodes can not sense the channel while transmitting (hence, are not aware of other ongoing transmissions). We assume that time is slotted into fixed length time frames. In any slot, a node having a packet to be transmitted to one of its neighboring devices decides with some fixed (possibly node dependent) probability in favor of a transmission attempt. If there is no other transmission by the other devices whose transmission can interfere with the node under consideration, the transmission is successful. As examples of this mechanism, we find Aloha-type [14] and IEEE 802.11 CSMA/CA-based mechanism. With these mechanisms, each node determines its transmission times [4,25]. In the UMTS network, the nodes communicate using Direct Sequence-Code Division Multiple Access (DD-CDMA). The node transmits the packet with fixed rate and fixed power [26]. We consider an automatic-repeat-request(ARQ) mechanism in which the node keeps retransmitting a packet until the packet is received at base station without any error.

At any instant in time, a device may have two kinds of packets to be transmitted:

1. Packets generated by the device itself. This can be sensed data if we are considering a sensor network.

2. Packets from other neighboring devices that need to be forwarded. 
We consider two separate queues for these two types and do a weighted fair queueing (WFQ) for these two queues. This configuration allows nodes to have flexibility for managing at each node forwarded packets and its own packets differently. Two queues allow to model the selfish behavior or on the contrary to give higher priority to connections that traverse many hops that could otherwise suffer from large loss rates. The main contribution of this paper is to provide approximation expressions of stability. Our main result is concerned with the stability of the forwarding queues at the devices. It states that whether or not the forwarding queues can be stabilized (by appropriate choice of WFQ weights) depends only on the routing and the channel access rates of the devices. The end-to-end throughput achieved by the nodes are independent of the choice of the WFQ weight. The context of the stability that we study is new as it takes into account the possibility of a limited number of transmissions of a packet at each node after which it is dropped.

Related works. Multihoming has been traditionally used by stub networks for improving network availability. Previous studies [5], have shown that "Multihoming Route Control" can improve the Internet communication performance of the networks significantly. A comparison study of overlay source routing and multihoming is presented in [6]. The goal of this work is how much benefit does overlay routing provides over BGP, when multihoming and route control are considered. In wireless network, the usefulness of attaching to the Internet using different access technologies at different time, or even simultaneously, has been described in various works [7,8]. A work devoted to IEEE 802.11 WLANs is [9], where users can choose between several access points and even split their traffic by using several of them. The network stability has been studied extensively both for networks with centralized scheduling [15, 17, Aloha protocol [10,21] and UMTS system [19,20]. Among the most studied stability problems are scheduling [15,16] as well as for the Aloha protocol [27, 24. Tassiulas and Ephremides [15] obtain a scheduling policy for the nodes that maximizes the stability region. Their approach inherently avoids collisions which allows to maximize the throughput. Radunovic and Le Boudec [11] suggest that considering the total throughput as a performance objective may not be a good objective. Moreover, most of the related studied do not consider the problem of forwarding and each flow is treated similarly (except for Radunovic and Le Boudec [11] or Tassiulas and Sarkar [23]).

The rest of the paper is organized as: In section II, we formulate the problem. In section III, we study the stability of any given node in the system using the rate balance equation. We analyze the uplink case in section IV, valid our model and provide some numerical examples and some concluding remarks in section $\mathrm{V}$.

\section{Model Formulation}

Background. Consider a geographical area which is not totally covered by any $3 \mathrm{G}$ system. The system is composed of three geographical classes (See fig 1); Class $\mathcal{C}_{1}$ contains terminals which communicate with each other using an ad-hoc network and some of them could be covered by the UMTS. The class $\mathcal{C}_{3}$ contains 
all terminals which are in the direct range of the $3 \mathrm{G}$ Node-B. We denote by $\mathcal{C}_{2}$ the set of mobiles covered by both the $3 \mathrm{G}$ system and the ad-hoc network, i.e. $\mathcal{C}_{2}=\mathcal{C}_{1} \cap \mathcal{C}_{3}$. Let $N_{1}=\operatorname{card}\left(\mathcal{C}_{1} \backslash \mathcal{C}_{2}\right)$ and $N_{2}=\operatorname{card}\left(\mathcal{C}_{2}\right)$. The $3 \mathrm{G}$ base station is denoted by $B$. The idea behind this work is to find a solution that allows non covered mobiles $\left(\mathcal{C}_{1} \backslash \mathcal{C}_{2}\right)$ to use the $3 \mathrm{G}$ services besides they are not in the covered area, and allow to $3 \mathrm{G}$ devices to connect to Internet thanks to ad-hoc network. So we consider a scheme with special kind of terminals, each one disposes of two network cards (IEEE 802.11 and DS-CDMA), hence it could be connected to either the ad-hoc or the $3 \mathrm{G}$ system according to the coverage criterion. Moreover this mobile is assumed to be able to forward packets arriving from ad-hoc mobiles, and therefore transmits them to the $3 \mathrm{G}$ node- $\mathrm{B}$ or to another ad-hoc node; In other terms these type of mobiles can play the role of gateways that allow to access to the node-B and therefore to benefit from its services.

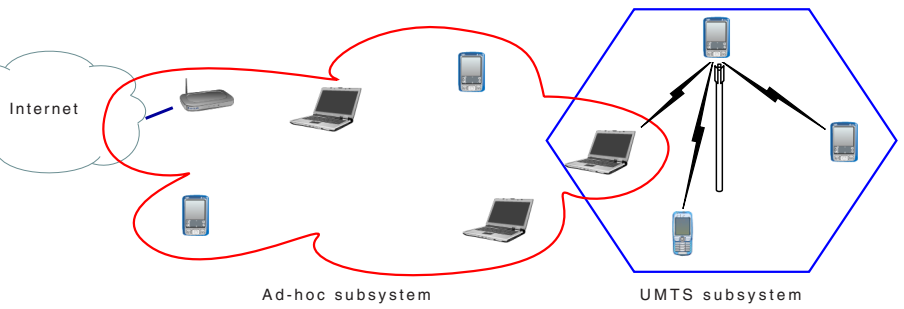

Fig. 1. A single Cell 3G system combined with ad-hoc network. Access to UMTS and Internet without coverage constraint using an IEEE 802.11/UMTS gateway solution.

As long as a $3 \mathrm{G}$ node is far of the base station as its transmit power is higher, which could deteriorate the other nodes QoS. To avoid this, far nodes will prefer to use their ad-hoc neighbors to transmit and therefore save their energy. Moreover, for a $3 \mathrm{G}$ user who needs to connect to the Internet, it is very interesting to access to it via ad-hoc for high rate and low cost comparing to Internet via UMTS.

Remark. (Case of a node $j \in \mathcal{C}_{2}$ ). Node $j$ has neighbors transmitting using the IEEE 802.11 band and others using the UMTS band; When $j$ transmits over the ad-hoc subsystem, it only collides with its neighbors using ad-hoc. And it is influenced only by users communicating with the node-B when it transmits using DS-CDMA. In others terms the two subsystems are separated and do not interfere with each other.

Network layer. In order to insure the forwarding of a packet to a given destination (ad-hoc node or the Node-B), we assume that each terminal $i$ possesses two queues $Q_{i}$ and $F_{i} . F_{i}$ is selected with probability $f_{i}$ and receives packet to be forwarded to another destination whereas $Q_{i}$ represents the queue which carries the own packets of node $i$ and is selected for transmission with probability $1-f_{i}$. In this work we consider the saturated case where node $i$ has always 
packets to transmit from $Q_{i}$. These queues are assumed to have infinite capacity. The network layer handles these queues using a weighted fair queuing (WFQ) protocol. For notation, we denote by $R_{s, d}$ the set of nodes between a source $s$ and a destination $d$ ( $s$ and $d$ not included).

MAC layer. The MAC layers of the two subsystems are different. We consider for ad-hoc subsystem a collision channel where no more than one node can transmit successfully in a slot; Denote by $\tau^{a}$ the duration of one slot. A successful transmission is occurred when there is no collision on reception, in other terms neighbors of an intermediate node should not transmit when it receives a packet. Each mobile transmitting a packet either from $Q_{i}$ or $F_{i}$ should access the channel by probability $P_{i}$. We can consider the aloha protocol, CSMA/CA or any other mechanism to access the channel. For instance, the attempt rate (for node $i$ ) in IEEE 802.11 DCF (see 25]) is,

$$
P_{i}=\frac{2\left(1-2 P_{c}\right)}{\left(1-2 P_{c}\right)\left(C W_{\min }+1\right)+P_{c} C W_{\min }\left(1-\left(2 P_{c}\right)^{m}\right)}
$$

$P_{c}$ denotes the conditional collision probability given that a transmission attempt is made and $C W$ is the contention window. $m=\log _{2}\left(\frac{C W_{\max }}{C W_{\min }}\right)$ is the maximum of backoff stage. We assume that a packet is retransmitted (if needed) till success or dropping, Let $K_{i, s, d}$ be the maximum number of transmissions allowed by a mobile $i$ per packet. Yet expression (1) is valid only for IEEE 802.11 systems. In our case we have an hybrid network, so it does not hold anymore and we shall calculate it. Assume $x_{i}$ is the total proportion of UMTS cycles for a given node $i$. Let $\tau_{i}^{u}$ be the average needed number of slots to send a UMTS packets.

Proposition 1. Consider a heterogenous network composed of a UMTS and an Ad-hoc subsystems, then we have the following,

1) The proportion of UMTS traffic is,

$$
x_{i}=P_{i, B}\left(1-\pi_{i} f_{i}\right)+f_{i} \sum_{s} \pi_{i, s, B}
$$

2) The attempt rate for any given node $i$ is,

$$
\bar{P}_{i}=\frac{\bar{L}_{i}\left(1-x_{i}\right)}{\bar{L}_{i}\left(1-x_{i}\right)+\tau_{i}^{u} x_{i} P_{i}} P_{i}
$$

Proof. Here we develop a cycle-based method to prove proposition [1 Let us observe the system (in particular a node $i$ ) for $C_{i, t}$ cycles. $C_{i, t}^{a}$ is the number of Ad-hoc cycles until the $t^{t h}$ slot, and $C_{i, t}^{u}$ is the corresponding number of cycles for the UMTS traffic. We denote by $T_{t}^{a}$ the total number of transmission slots used by Ad-hoc connections till the $t^{t h}$ slot.

The proportion of UMTS cycles is $x_{i}=\frac{C_{i, t}^{u}}{C_{i, t}}=\frac{C_{i, t}^{u, Q}+C_{i, t}^{u, F}}{C_{i, t}}$ 
$\frac{C_{i, t}^{u, Q}}{C_{i, t}}$ is the probability to choose a UMTS packet from $Q_{i}$. We have

$\frac{C_{i, t}^{u, Q}}{C_{i, t}}=\frac{C_{i, t}^{u, Q}}{C_{i, t}^{Q}} \cdot \frac{C_{i, t}^{Q}}{C_{i, t}}=P_{i, B}\left(1-\pi_{i} f_{i}\right)$

$\frac{C_{i, t}^{u, F}}{C_{i, t}}=\frac{C_{i, t}^{F}}{T_{i, t}^{a}} \cdot \frac{T_{i, t}^{a}}{C_{i, t}} \cdot \frac{C_{i, t}^{u, F}}{C_{i, t}^{F}}=f_{i} \pi_{i} \sum_{s} \frac{\pi_{i, s, B}}{\pi_{i}}=f_{i} \sum_{s} \pi_{i, s, B}$

where $T_{i, t}^{a}$ denotes the number of cycles having Ad-hoc packets till slot $t$.

The proportion of UMTS cycles becomes $x_{i}=P_{i, B}\left(1-\pi_{i} f_{i}\right)+f_{i} \sum_{s} \pi_{i, s, B}$ The proof is complete for part 1 .

The long term attempt rate is $\bar{P}_{i}=\lim _{t \rightarrow \infty} \frac{T_{t}^{a}}{t}=\lim _{t \rightarrow \infty} \frac{T_{t}^{a}}{C_{i, t}^{a}} \cdot \frac{C_{i, t}^{a}}{C_{i, t}} \cdot \frac{C_{i, t}}{t}$ $\lim _{t \rightarrow \infty} \frac{T_{t}^{a}}{C_{i, t}^{a}}$ is the average number of slots per cycle (Ad-hoc or UMTS), i.e. $\bar{L}_{i}$. $\lim _{t \rightarrow \infty} \frac{C_{i, t}^{a}}{C_{i, t}}$ is exactly the proportion of Ad-hoc cycles among $C_{i, t}$, i.e. $1-x_{i}$. $\lim _{t \rightarrow \infty} \frac{t}{C_{i, t}}$ is the average number of slots per cycle (Ad-hoc or UMTS), then $\lim _{t \rightarrow \infty} \frac{t}{C_{i, t}}=\frac{C_{i, t}^{a} \cdot \frac{\bar{L}_{i}}{P_{i}}+C_{i, t}^{u} \cdot n}{C_{i, t}}=\frac{\bar{L}_{i}}{P_{i}}\left(1-x_{i}\right)+\tau_{i}^{u} x_{i}$

The result follows by substituting each term by its established expression.

For the UMTS subsystem, we assume that the base station communicates with 3G nodes using Direct Sequence Code Division Multiple Access (DS-CDMA). Each user $i$ transmits to the base station with a transmission rate $\rho_{i, B}$ (Uplink transmission rate), which is the bandwidth to the processing gain ratio. It depends on the mobile classes, the solicited service and the global load. The base station uses a downlink transmission rate $\rho_{B, i}$ when sending to $i$. Another important parameter is the Packet Success Rate (PSR), which we denote by $\xi\left(\gamma_{i}\right)$, it is a function of the instantaneous signal to noise plus interference ratio (SINR). $\xi($.$) could be any increasing continuous, differentiable and S-sharped$ (sigmoidal) function with $\xi(0)=0$ and $\xi(+\infty)=1$. Some properties and examples of S-sharped functions are studied with more details in [26] and references therein.

Define $\mu_{i, B}$ as the uplink service rate, i.e. at which UMTS packets are served at node $i$. We denote by $\mu_{B, i}$ the arrival rate to node $i$ from the node-B. Let $\tau_{i, B}=\frac{1}{\mu_{i, B}}$ be the average service time in number of ad-hoc slots. Then, it can be written as: $\tau_{i, B}=\left\lceil\frac{M_{a}}{\rho_{i, B} \xi\left(\gamma_{i}\right) \cdot \tau^{a}}\right\rceil$ where $M_{a}$ is the length of one ad-hoc packet (in bit) and $\frac{M_{a}}{\rho_{i, B} \xi\left(\gamma_{i}\right)}$ is the average service time in second of a packet while allowing infinity retransmissions of a failure packet, see [26].

Cross-layer architecture. Nodes with two network cards are able to communicate with other nodes or with the $3 \mathrm{G}$ node-B. The system performance depends on more than one layer, this motivates us to use a cross-layer architecture (fig 2). This new network insight is more powerful and flexible thanks to communication and information exchange between different layers.

Remark. It is interesting to note that a mobile is able to transmit on one interface and receive on the other and vice-versa. Moreover, it could get si- 


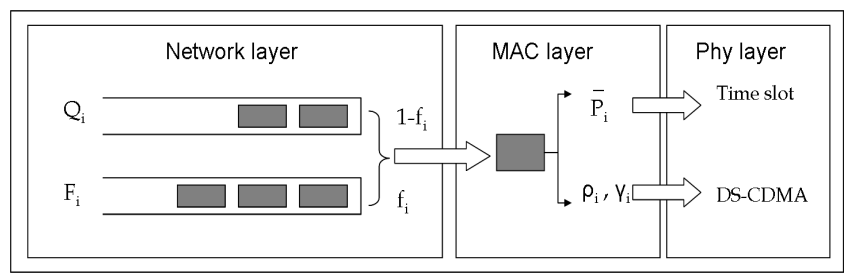

Fig. 2. Cross-layer architecture including the two subsystems

multaneously one ad-hoc and one UMTS reception. Whereas we do not allow simultaneous transmissions over the two network cards. This is due to the nature of scheduling mechanism that we consider (selection of one queue a time). If we allow parallel selections we could make two simultaneous transmissions possible.

\section{$3 \quad$ Stability of Forwarding Queues}

In this section we study stability properties of nodes in the system in particular. a node $i \in \mathcal{C}_{2}$ which we call gateways. Classically , the forwarding queue $F_{i}$ is stable if the departure rate of packets from $F_{i}$ is at least equal to the arrival rate into it. This is expressed by a rate balance system. For any given nodes $i, s, d \in \mathcal{C}_{1} \cup \mathcal{C}_{3} ; i$ denotes an intermediate node, $s$ is the source and $d$ is the destination. Let $j_{i, s, d}$ denote the entry in the set $R_{s, d}$ just after $i$ and $\mathcal{N}(i)$ be the set of $i$ 's neighbors. The probability that a transmission from node $i$ on route from node $s$ to node $d$ is successful is,

$$
P_{i, s, d}=\prod_{j \in j_{i, s, d} \cup \mathcal{N}\left(j_{i, s, d}\right) \backslash i}\left(1-\bar{P}_{j}\right)
$$

The expected number of attempts till success or dropping from $i$ on route $R_{s, d}$ is,

$$
L_{i, s, d}=\frac{1-\left(1-P_{i, s, d}\right)^{K_{i, s, d}}}{P_{i, s, d}}
$$

Let $\pi_{i, s, d}$ be the probability that the queue $F_{i}$ has a packet at the first position ready to be forwarded to the path $R_{s, d}$. It follows that the probability that the queue $F_{i}$ has at least one packet to be forwarded is $\pi_{i}=\sum_{s, d} \pi_{i, s, d}$. If $P_{i, d}$ denotes the probability that $i$ transmits to $d$ then the average of $L_{i, s, d}$ over all possible paths is,

$$
\bar{L}_{i}=\sum_{s, d: i \in R_{s, d}} \pi_{i, s, d} f_{i} L_{i, s, d}+\sum_{d}\left(1-\pi_{i} f_{i}\right) P_{i, d} L_{i, i, d}
$$

In the following we classify connections onto three categories.

- ad-hoc to ad-hoc connections: This corresponds to the case studied in 1 in which an ad-hoc node transmits to another ad-hoc node, i.e. $s, d \in \mathcal{C}_{1}$. 
- ad-hoc to UMTS connections: This is the uplink case in which nodes transmit to the base station. For $s \in \mathcal{C}_{1}$ and $d=B$, note that a UMTS packet is transmitted until success from the gateway to the base station.

- UMTS to ad-hoc connections: This illustrates downlink scheme, in other terms when the base station is transmitting to a given node so $s=B$ and $d \in \mathcal{C}_{1} \cup \mathcal{C}_{3}$.

We note for transmissions to the base station, that an intermediate node $i$ forwards packets to a next hop at a rate $\frac{P_{i}}{\bar{L}_{i}}$, whereas it forwards them to the base station (if it is in the direct range of $\mathrm{B}$ ) at a rate $\mu_{i, B}$. For more generality we define $\bar{\mu}_{i, B}$ as the service rate for the uplink scheme as following,

$$
\bar{\mu}_{i, B}= \begin{cases}\mu_{i, B}, & \text { if } \quad i \in \mathcal{C}_{3} \\ \frac{P_{i}}{\bar{L}_{i}}, & \text { if } \quad i \in \mathcal{C}_{1} \backslash \mathcal{C}_{2}\end{cases}
$$

\section{The departure rate}

In order to compute the departure rate of a given node, we shall make differentiation between ad-hoc and UMTS connections. Also the geographical criterion is taken into consideration thanks to $\bar{P}_{i}$ and $\bar{\mu}_{i, B}$.

The long term departure rate from node $i$ for the connection $R_{s, d}$ is,

$$
d_{i, s, d}=\left\{\begin{array}{lr}
\pi_{i, s, d} f_{i} \frac{\bar{P}_{i}}{\bar{L}_{i}}, & s, d \in \mathcal{C}_{1} \\
\pi_{i, s, B} f_{i} \bar{\mu}_{i, B}, & s \in \mathcal{C}_{1}, d=B \\
\pi_{i, B, d} f_{i} \frac{\bar{P}_{i}}{\bar{L}_{i}}, & d \in \mathcal{C}_{1}, s=B
\end{array}\right.
$$

\section{The arrival rate}

Here, we compute the arrival rate for each node $i$. Let $i, s, d \in \mathcal{C}_{1} \cup \mathcal{C}_{3}$. $s$ sends a packet destined to $d$ with probability $P_{s, d}$. When the UMTS node-B transmits to a node $d$, we denote by $g$ (for gateway) the first hop on the route $R_{B, d}$. $i$ 's long term arrival rate is expressed, according to possible connections, as following:

$$
a_{i, s, d}=\left\{\begin{array}{lr}
\left(1-\pi_{s} f_{s}\right) \frac{\bar{P}_{s}}{\bar{L}_{s}} P_{s, d} \prod_{k \in R_{s, i} \cup s}\left[1-\left(1-P_{k, s, d}\right)^{K_{k, s, d}}\right], & s, d \in \mathcal{C}_{1} \\
\left(1-\pi_{s} f_{s}\right) \frac{\bar{P}_{s}}{\bar{L}_{s}} P_{s, B} \prod_{k \in R_{s, i} \cup s}\left[1-\left(1-P_{k, s, B}\right)^{K_{k, s, B}}\right], & s \in \mathcal{C}_{1}, d=B \\
\mu_{B, g} \prod_{k \in R_{B, d}}\left[1-\left(1-P_{k, s, d}\right)^{K_{k, s, d}}\right], & d \in \mathcal{C}_{1}, s=B
\end{array}\right.
$$

End to end throughput of a connection. The end to end throughput between a couple of nodes $s$ and $d$ is exactly the arrival rate to the destination $d$. Namely $t h p_{s, d}=a_{d, s, d}$. Recall that gateways keep transmission of a packet over the UMTS subsystem till success (correctly received by the base station). 


\subsection{The Rate Balance System (RBS)}

A queue $F_{i}$ is stable while its corresponding departure rate is greater or equal than the arrival rate into it. In this subsection we consider the extreme case where we have strict equality. We will derive the rate balance equation for every node in the system.

When the steady state is achieved, if all queues are stable, then for each $i, s$ and $d$ such that $i \in R_{s, d}$ we get $d_{i, s, d}=a_{i, s, d}$, this is the rate balance equation on the path $R_{s, d}$. Then for all $i, s$ and $d$, we get a system of equations from which we can find a solution of the $\pi_{i, s, d}$ for all $i, s$ and $d$. And then, find $\pi_{i}$, for all $i$, which determines the load of the forwarding queues and the main condition of their stability which is $\pi_{i}<1$. Each node can forward packets for the three types of connections defined previously, and therefore we can explicit the rate balance equations using three generic equations:

$$
\begin{cases}\pi_{i, s, d} f_{i}=\left(1-\pi_{s} f_{s}\right) \frac{\bar{P}_{s} \bar{L}_{i}}{\bar{P}_{i} P_{s}} P_{s, d} \prod_{k \in R_{s, i} \cup s}\left[1-\left(1-P_{k, s, d}\right)^{K_{k, s, d}}\right], & s, d \in \mathcal{C}_{1} \\ \pi_{i, s, B} f_{i}=\frac{\left(1-\pi_{s} f_{s}\right)}{\bar{\mu}_{i, B}} \frac{\bar{P}_{s}}{\bar{L}_{s}} P_{s, d} \prod_{k \in R_{s, i} \cup s}\left[1-\left(1-P_{k, s, d}\right)^{K_{k, s, d}}\right], & s \in \mathcal{C}_{1}, d=B \\ \pi_{i, B, d} f_{i}=\mu_{B, g} \frac{\bar{L}_{i}}{\bar{P}_{i}} \prod_{k \in R_{B, d}}\left[1-\left(1-P_{k, s, d}\right)^{K_{k, s, d}}\right], & d \in \mathcal{C}_{1}, s=B\end{cases}
$$

We sum over all sources $s$ and destinations $d$ for all connections written above and get the global rate balance equation which is useful to study some special cases.

Theorem 1. In the steady state, if all the queues in the system (both ad-hoc and UMTS) are stable, then for each $i, s, d \in \mathcal{C}_{1} \cup \mathcal{C}_{3}$, such that $i \in R_{s, d}$ we have,

$$
\begin{aligned}
& \frac{\bar{P}_{i} f_{i}}{\bar{L}_{i}} \sum_{s, d} \pi_{i, s, d} f_{i}+\bar{\mu}_{i, B} \sum_{s} \pi_{i, s, B}=\sum_{d: i \in R_{B, d}} \mu_{B, i} \prod_{k \in R_{B, i}}\left[1-\left(1-P_{k, B, d}\right)^{K_{k, B, d}}\right] \\
& +\sum_{s, d: i \in R_{s, d}}\left(1-\pi_{s} f_{s}\right) \frac{\bar{P}_{s} P_{s, d}}{\bar{L}_{s}} \prod_{k \in R_{s, i} \cup s}\left[1-\left(1-P_{k, s, d}\right)^{K_{k, s, d}}\right]
\end{aligned}
$$

Let $z_{i, s, d}=\pi_{i, s, d} f_{i}$, for all $i, s$ and $d$, be the unknown of the rate balance system which is a non linear system because $\bar{P}_{i}$ and $\bar{L}_{i}$ usually depend on $z_{i, s, d}$. We remark as in paper [1] that the solution is independent on the forwarding probability, and consequently the end to end throughput is also not impacted by the choice of $f_{i}$ as it appears when computing the end-to-end throughput $t h p_{s, d}=a_{d, s, d}$. This result holds only when the forwarding queues are stable. As a consequence, the forwarding capabilities of gateways do not affect their energy consumption. 
Another important remarks concern the interaction between the ad-hoc and the UMTS. Practically, the bit rate per node in the ad-hoc network is larger than the UMTS, but the size of this former network and its characteristics (as channel access and routing) influence drastically its capacity. Yet, connecting two heterogenous networks normally needs a scaling and an adaptation of the rates on each interface, if not, stability of the gateway nodes becomes a real issue.

From the rate balance system, we find $z_{i, s, d}$ function of the service rate of the UMTS node $\bar{\mu}_{i, B}$ and of the base station rate $\mu_{B, i}$. So, the condition on $z_{i, s, d}$ $\left(z_{i, s, d}<1\right)$ will be translated to a condition on the parameters of the UMTS, for example, we can find a minimum packets rate for UMTS nodes or a threshold of the SINR $\gamma$ that insures stability on gateway nodes.

Concerning the throughput of ad-hoc nodes connected to the base station, the throughput can be written in a simple form, in case of stability, as: $\operatorname{th} p_{s, B}=$ $z_{g, s, B} \mu_{g, B}$, where $g$ is a gateway node. For the particular uplink case, the arrival rate (second equation of (8) ) is independent of $\mu_{i, B}$ since $\pi_{s} f_{s}, L_{i}$ and $P_{i}$ do not depend on it. Then when $\mu_{i, B}$ changes the load $z_{i, s, B}$ changes in the opposite sense, it follows that the throughput stays unchanged.

\section{The Uplink Case Analysis}

In the following we discuss and study with more details a scheme when the RBS is linear such the uplink case which is very important. There is indeed many asymmetric services which do not requires any request-response pattern (e.g. downloading files). In this case all sources has the same destination and each intermediate node forwards always to the same next hop. it follows that $\bar{L}_{i}$ is independent of the unknown $\pi_{i}$,

$\bar{L}_{s}=\sum_{s^{\prime}} \pi_{s, s^{\prime}, d} f_{s} L_{s, s^{\prime}, d}+\left(1-\pi_{s} f_{s}\right) P_{s, d} L_{s, s, d}=\pi_{s} f_{s} L_{s, s, d}+\left(1-\pi_{s} f_{s}\right) L_{s, s, d}=L_{s, s, d}$

The RBS is reduced to the second equation when $d=B$, we sum over all $s$,

$$
\sum_{s} z_{i, s, B}=\sum_{s} \frac{y_{s} \bar{P}_{s} P_{s, B}}{\bar{\mu}_{i, B} \bar{L}_{s}} \prod_{k \in R_{s, i} \cup s}\left[1-\left(1-P_{k, s, d}\right)^{K_{k, s, d}}\right]
$$

Let $y_{i}=1-\pi_{i} f_{i}$, later we will refer to $y_{i}$ as the stability region of $F_{i}$. We have,

$$
y_{i}+\sum_{s} y_{s} \omega_{i, s}=1
$$

where $\omega_{i, s}=\sum_{d} \frac{\bar{P}_{s} P_{s, d}}{\bar{\mu}_{i, B} \bar{L}_{s}} \prod_{k \in R_{s, i} \cup s}\left[1-\left(1-P_{k, s, d}\right)^{K_{k, s, d}}\right]$. (11) can be written in a matrix form and then will be resolved more easily.

$$
\underline{Y}(I+\bar{W})=\underline{1}
$$


$\bar{W}$ is the $N \times N$ matrix whose $(s, i)^{t h}$ entry is $\omega_{s, i}, \underline{Y}$ is the unknown $N$-dimensional row vector, i.e. $Y(i)=y_{i}$.

In the following we will restrict to symmetric ad-hoc network. The transmit and forwarding probabilities are the same for all users, i.e. $P_{i}=P$ and $f_{i}=f$. Assume that every ad-hoc node $i$ has the same number of neighbors $n_{i}=n$ (such a grid or a mesh), it follows that $P_{i, s, B}=(1-P)^{n}$. For simplification and without loss of generality we will be assuming that $K_{i, s, d} \equiv 1$. Let denote by $d(i, s, B)$ the number of intermediate nodes on the route $R_{s, B}$. Since we only consider uplink connections we shall put $\mu_{B, g}=0$.

Proposition 2. A necessary condition for stability of $F_{i}$, for any given node in the system, for the uplink scheme is that,

$$
f \geq \frac{\bar{\omega}_{i, s, B}}{\bar{\omega}_{i, s, B}+\bar{\mu}_{i, B}} \quad \text { s.t. } \quad f \leq 1
$$

where $\bar{\omega}_{i, s, B}=\sum_{s} P(1-\bar{P})^{n(d(i, s, B)+1)}$

Proof. For any given routing, the input ratio into the forwarding queue $F_{i}$ is,

$$
\begin{aligned}
a_{i} & =\sum_{s}\left(1-f \pi_{s}\right) P P_{s, s, B} \prod_{k \in R_{s, i}}\left[1-\left(1-P_{k, s, B}\right)^{K_{k, s, B}}\right] \\
& \geq \sum_{s}(1-f) P(1-\bar{P})^{n(d(i, s, B)+1)}=a_{i}^{\text {min }}
\end{aligned}
$$

The departure rate is $\quad d_{i}=\bar{\mu}_{i, B} f \pi_{i} \leq \bar{\mu}_{i, B} f=d_{i}^{\max }$

Since $F_{i}$ is stable if and only if $d_{i} \geq a_{i}$, then the result follows.

The next corollary provides a condition related to UMTS subsystem.

Corollary. Considering the uplink case, a necessary condition that insures stability of queue $F_{i}$ for any given gateway $i$, is that,

$$
\mu_{i, B} \geq \frac{1-f}{f} \cdot \bar{\omega}_{i, s, B}
$$

A more useful form is to convert it onto a condition on instantaneous SINR as,

$$
\gamma_{i} \geq \xi^{-1}\left(\frac{1-f}{f} \cdot \frac{M_{a}}{\rho_{i, B} \tau^{a}} \cdot \bar{\omega}_{i, s, B}\right)
$$

where $\bar{\omega}_{i, s, B}=\sum_{s} P(1-\bar{P})^{n(d(i, s, B)+1)}$. An interesting result of proposition (13) is that UMTS parameters do not affect stability of non covered nodes.

\section{Numerical Results and Simulations}

Consider in fig. (3) one single UMTS cell, covered by base station $B$, and extended with an Ad-Hoc network which is itself related to Internet via AP 9. 


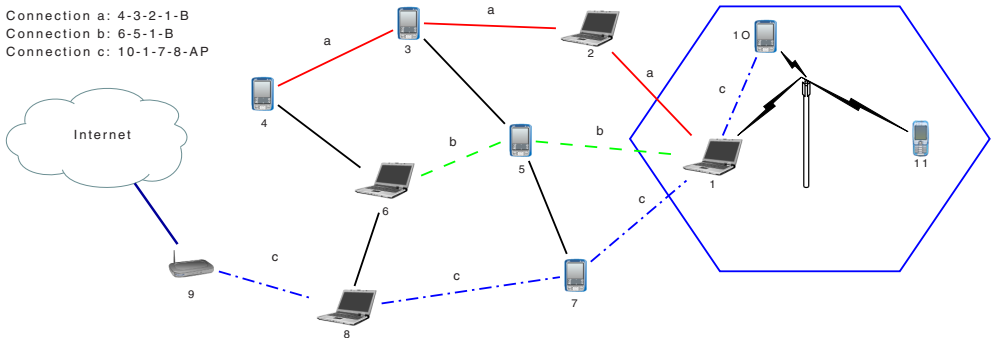

Fig. 3. Cellular and Ad-Hoc networks
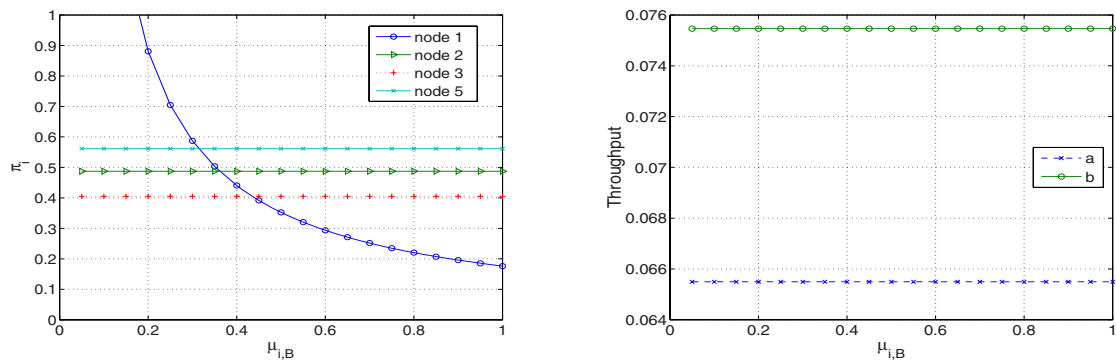

Fig. 4. Queues load for $K=4$ and $f=0.8$ Fig. 5. Throughput of connections $a$ and $b$

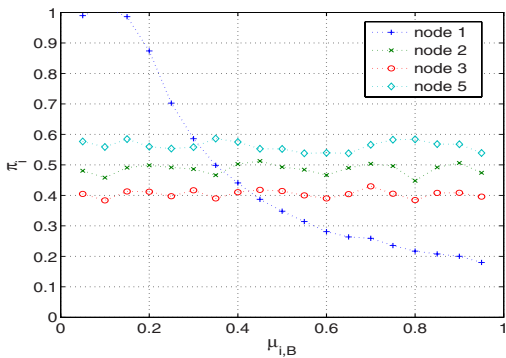

Fig. 6. Queues load $\pi_{i}$ from simulation

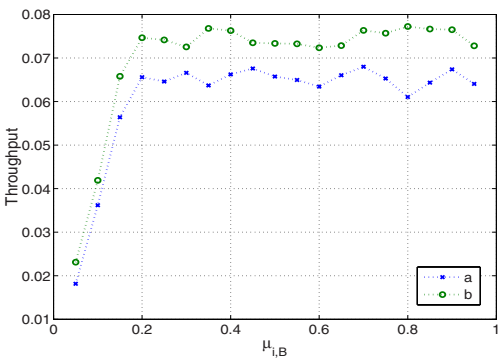

Fig. 7. Throughput from simulation

Both nodes in the UMTS cell and the Ad-Hoc network can access to the services of the two networks. For that, gateway nodes play an important relay role on forwarding packets for both technologies. For that we are interested to show, at first, numerical results concerning the load of these latter nodes and then the end -to-end throughput of connections that traverse these nodes. We use a discrete time simulator that reflects the behavior of nodes in our model described in section 2, to validate our formulation results.

In fig. (3), nodes 4 and 6 access the UMTS system with the base station $B$ via connections $a$ and $b$. Node 10 which is in the UMTS cell also reaches the access point AP (node 9) via the Ad-Hoc network with a connection $c$. 
We fixe $P_{3}=P_{9}=0.2, P_{4}=0.4$ and all other $P_{i}$ to 0.3 . The number of transmissions is set to $K_{i, s, d} \equiv K=4$ and the forwarding probability to $f_{i} \equiv f=0.8$. Fig. (4) and (5) consider only the uplink case where Ad-Hoc nodes communicate with the base station. Fig. 4 shows the load variation function of the UMTS service rate $\mu_{1, B}$. It is clear that increasing $\mu_{1, B}$ would ameliorate the stability of node 1 . Here, we see that we need $\mu_{1, B}>0.19$ to get $\pi_{1}<1$. For an Ad-Hoc node rate of 2Mbps and an Ad-Hoc packet size of 1000 Bytes, the slot size becomes $\tau^{a}=4 \mathrm{~ms}$, then a minimum UMTS rate needed must be at least 47.5 packet/s (ad-hoc packet). Concerning the throughput, fig. (5) shows the insensitivity of the throughput with the service rate of the UMTS. Note that only for $\pi_{1}<1$ the figure of the throughput make sense. Elsewhere it will be an increasing function of $\mu_{1, B}$ as it is shown in the simulation fig. (7). A validation with simulations of the numerical results is shown in fig. (7]6).

Conclusion. In this paper we propose extending the coverage of UMTS using an ad-hoc network, our main result is characterization of stability condition and the end-to-end throughput, we also validate the analytical result using a discrete simulator.

An ongoing work consists on studying the impact of transmit power over the $3 \mathrm{G}$ subsystem on the stability of the forwarding queues. We also consider a joint power and rate control game to seek the equilibrium points under some QoS constraints.

\section{References}

1. El Khoury, R., Elazouzi, R.: Stability-throughput analysis in a multi-hop ad hoc networks with weighted fair queueing. In: The Proc. 45th Annual Allerton Conf. on Communication, Control, and Computing (Allerton 2007), Monticello, IL (September 2007)

2. Kherani, A., ElAzouzi, R., Altman, E.: Stability-Throughput tradeoff and routing in multi-hop wireless ad-hoc networks. In: The Proceeding of Networking Conference 2006, Coimbra, Portugal, MAY 19, vol. 15 (2006) (Best paper Award)

3. Bria, A.: Joint resource management of cellular and broadcasting systems-research challenges. In: RVK 2005 Conference (2005)

4. Bianchi, G.: Performance analysis of the IEEE 802.11 distribute coordination function. IEEE Journal on Selected Areas in Communications (March 2000)

5. Smith, P.: Bgp multihoming techniques. In: NANOG, vol. 23 (2001)

6. Shaikh, A., Sitaraman, R., Akella, A., Maggs, B.: A measurement-based analysis of multihoming. In: SIGCOMM (2003)

7. Stemm, R., Katz, R.: Vertical handoffs in wireless overlay networks. Journal on Mobile Networks and applications 3(4), 335-350 (1998)

8. Wakikawa, R., Paik, E.K., Ng, C.W., Ernst, T., Montavont, N., Noel, T.: Goal and benefits of multihoming. IETF Internet Draft (July 2005)

9. Altman, E., Shakkottai, S., Kumar, A.: The case for non-cooperative multihoming of users to access points in ieee 802.11 wlans. In: IEEE INFOCOM 2006, Barcelona (2006)

10. Rao, R., Ephremides, A.: On the stability of ineracting queues in a multi-access system. IEEE Tran. Inform. Theory 34, 918-930 (1988) 
11. Radunovic, B., Le Boudec, J.Y.: Joint Scheduling, Power Control and Routing in Symmetric, One-dimensional, Multi-hop Wireless Networks. In: WiOpt 2003: Modeling and Optimization in Mobile, Ad-Hoc and Wireless Networks, SophiaAntipolis, France (2003)

12. Clausen, T., Jacquet, P., Laouiti, A., Muhlethaler, P., Qayyum, A., Viennot, L.: Optimized Link State Routing Protocol. In: IEEE INMIC Pakistan (2001)

13. Murthy, S., Garcia-Luna-Aceves, J.J.: An Efficient Routing Protocol for Wireless Networks. ACM/Baltzer Journal on Mobile Networks and Applications, Special Issue on Routing in Mobile Communication Networks 1 (1996)

14. Roberts, L.G.: Aloha packet system with and without slots and capture, Tech. Rep. Ass Notes stanford Research Institute advance Recharch Projects Agency Network Infomation Center (1972)

15. Tassiulas, L., Ephremides, A.: Stability properties of constrained queuing systems and scheduling for maximum throughput in multihop radio network. IEEE Trans. Automat. Contr. 37(12), 1936-1949 (1992)

16. Tassiulas, L.: Linear complexity algorithm for maximum throughput in radio networks and input queued switches. In: IEEE Infocom 1998, pp. 533-539 (1998)

17. Tassiulas, L.: Scheduling and perfomance limits of networks with contantly changing topology. IEEE TRans. Inform. Theory 43(3), 1067-1073 (1997)

18. Altman, E., Borkar, V.S., Kherani, A.A., Michiardi, P., Molva, R.: Some game theoretic problems in wireless ad-hoc networks. In: Kotsis, G., Spaniol, O. (eds.) Euro-NGI 2004. LNCS, vol. 3427, pp. 82-104. Springer, Heidelberg (2005)

19. Schröder, B., Weller, A.: Prediction of the Connection Stability of UMTS Services in the downlink- an Analytical Approach. In: Vehicular Technology Conference (2002)

20. Kumar, A., Patil, D.: Stability and throughput analysis of unslotted CDMAALOHA with finite number of users and code sharing. Telecommunication Systems 8 (December (2-4), 1997)

21. Luo, W., Ephremides, A.: Stability of $\mathrm{N}$ interacting queues in multiqueue distributed systems: buffered random acess system. IEEE Trans. Inform. Theory 45, 1579-1587 (1999)

22. Tsybakov, B., Mikhailov, W.: Ergodicity of slotted Aloha system. Prob. Inform. Transmission 15(4), 301-312 (1979)

23. Tassiulas, L., Sarkar, S.: Max-Min fair scheduling in wireless networks. In: Proceeding of Infocom 2002 (2002)

24. Tsybakov, B.S., Bakirov, V.L.: Packet transmission in radio networks. Probl Infom. Transmission 21(1), 60-76 (1985)

25. Yang, Y., Hou, J.C., Kung, L.-C.: Modeling the effect of transmit power and physical carrier sense in multi-hop Wireless networks. In: Infocom 2007, Alaska (2007)

26. Meshkati, F., Poor, H.V., Schwartz, S.C., Mandayam, N.B.: An energy efficient approach to power control and receiver design in wireless data networks. IEEE transactions on communications 53(11), 1885-1894 (2005)

27. Anantharam, V.: The stability region of the finite-user slotted Alloha protocol. III Trans. Inform. Theory 37(3), 535-540 (1991) 